\title{
The Internal Coherence of Breast Cancer Patients Is Associated with the Decision-Making for Chemotherapy and Viscum album L. Treatment
}

\author{
Shiao Li Oei $\mathbb{D}^{1},{ }^{1}$ Anja Thronicke, ${ }^{1}$ Matthias Kröz, ${ }^{1,2,3,4}$ \\ Cornelia Herbstreit, ${ }^{2}$ and Friedemann Schad ${ }^{1 D}{ }^{1,2}$ \\ ${ }^{1}$ Research Institute Havelhöhe, D-14089 Berlin, Germany \\ ${ }^{2}$ Hospital Havelhöhe, D-14089 Berlin, Germany \\ ${ }^{3}$ Charité-Universitätsmedizin Berlin, Corporate Member of Freie Universität Berlin, Humboldt-Universität zu Berlin, \\ and Berlin Institute of Health, Germany \\ ${ }^{4}$ Institute for Integrative Medicine, University of Witten/Herdecke, D-58313 Witten/Herdecke, Germany \\ Correspondence should be addressed to Friedemann Schad; fschad@havelhoehe.de
}

Received 27 February 2018; Accepted 5 September 2018; Published 27 September 2018

Academic Editor: Mario Ledda

Copyright (c) 2018 Shiao Li Oei et al. This is an open access article distributed under the Creative Commons Attribution License, which permits unrestricted use, distribution, and reproduction in any medium, provided the original work is properly cited.

Objective. In the present observational study, the influence of internal coherence on shared decision-making for chemotherapy (CTX) and Viscum album L. extracts (VA) treatment in breast cancer patients was evaluated. Methods. Breast cancer patients with a guideline-oriented advice from the tumor board for CTX were included in the study. At first diagnosis (T0) and 6 months later (T1), a questionnaire, the internal coherence scale (ICS), was administered and evaluated. Prior to analysis, patients were classified retrospectively depending on their treatment decision. Results. 64 primary nonmetastasized breast cancer patients (median age 54.8 years, IQR: 46.3-65.3) were analyzed in this study. At T0, adjusted multivariable linear regression analyses revealed significant low ICS scores in patients rejecting CTX, especially in the ICS subscale "thermo coherence" $(p=0.006)$. The decision for add-on VAtherapy was associated with low scores for the ICS subscale "inner resilience coherence", in particular low for the item "courage". At T1, in the CTX+VA-group the thermo coherence increased significantly $(\mathrm{p}(\mathrm{d})<0.01)$, while in contrast, in the CTX-only group the thermo coherence decreased significantly $(\mathrm{p}(\mathrm{d})=0.02)$. Conclusion. Add-on VA-applications in CTX treatment support the thermo coherence of breast cancer patients, revealing a decision option to encourage patients to undergo CTX in combination with additional VA-treatments.

\section{Introduction}

Breast cancer is the most abundant malignant tumor in women worldwide [1]. Early detection methods and the establishment of improved treatment strategies reduced breast cancer mortality [2]. According to the German S3-guideline for early detection, diagnosis, therapy, and follow-up of breast cancer, adjuvant chemotherapy (CTX) is indicated for HER2-positive tumors, non-endocrine (or unclear) sensitive tumors, lymph node-positive disease, grade 3 tumors, luminal B tumors, and for patients aged $<35$ years [3]. Decisions about adjuvant CTX in women with early stage breast cancer are often challenging [4]. Uncertainty about benefits versus evolving side-effects complicates decision-making. The factors that influence the treatment decisions are complex and involve issues regarding access to health care, concerns for cancer recurrence, and the impact of cancer therapies on health-related quality of life (HRQL). HRQL has gained growing importance in clinical oncology $[5,6]$. Numerous questionnaires have been developed, to objectify and standardize HRQL analysis [7]; among them the mainly utilized is the European Organization for Research and Treatment of Cancer Quality of Life Questionnaire Core 30 (EORTC QLQ-C30) [8] and the Functional Assessment of Cancer Therapy (FACT) scale [9]. Beyond those captured dimensions of HRQL, other relevant aspects such as the sense of coherence [10,11] and self-regulation [12] are more appropriate as prognostic outcomes in long-term 
observational studies of cancer patients [13]. The sense of coherence seems to have a positive impact on the HRQL [14]. As a prognostic clinical tool in oncology, a specific 10 -item internal coherence scale (ICS) with self-reported questions capturing the individual skills of adaption, with good to very good reliability and sensitivity for treated cancer patients, was developed and validated [15]. During cancer treatment a comfort of well-being is often associated with good thermoregulation being a precondition on the subject of internal coherence $[16,17]$. Therefore, in the ICS, items on thermo coherence are integrated. The structure of this ICS enables us to measure the total-ICS score and the subscales "inner resilience and coherence" and "thermo coherence" [15]. Moreover, this questionnaire especially has a good responsiveness during CTX and shows some sensitivity for VA-treatment [18].

Women with breast carcinoma appear to have a strong desire for involvement in making decisions regarding their treatment [4]. Patient's expectations and motivations for using complementary alternative medicine are often chosen to manage pain or other treatment-related side-effects [19]. The feeling of being active involved in treatment decisions, with an associated level of self-control, is an important motivation for patients to use complementary alternative medicine [20]. Viscum album L. extracts (VA, mistletoe) are frequently used in integrative oncology to reduce treatmentrelated side-effects $[21,22]$. VA-applications are usually well tolerated and had only few and mild side-effects [23-25]. Various reports support clinical findings that VA-therapy can improve and stabilize HRQL in cancer patients, especially in breast cancer during CTX [26-29]. In this noninterventional, observational study, the ICS was used to evaluate the effects of add-on VA-therapy on the internal coherence in breast cancer patients with the advice for CTX. Firstly, the ICS at first diagnosis was analyzed to compare the individual internal coherence of the patients in regard to their decision for CTX and/or add-on VA-treatment. Furthermore, the change on the ICS, in particular the thermo coherence score of the patients during therapy, 6 months after first diagnosis was followed. In addition, occurring tumor recurrences within 3 years after first diagnosis were evaluated for the study group.

\section{Methods}

2.1. Study Design. We conducted a prospective observational study for primary stage I-III breast cancer patients as reported previously $[30,31]$. Patients who gave written consent to be registered in the Network Oncology (NO) registry [32], who were diagnosed between June 2012 and April 2017 at the certified Breast Cancer Centre (Gemeinschaftskrankenhaus Havelhöhe, Berlin, Germany), and who received a tumor board advice for CTX were included. Follow-up examinations were performed until December 2017 and only patients were included here from which completed ICS questionnaires at first diagnosis (T0) and 6 months after first diagnosis (T1) were available. Patients who received no advice for CTX were excluded. The NO registry has been approved by the ethics committee of the Medical Association Berlin (Eth-27/10).
2.2. Data Collection. The NO registry utilizes linked data from primary and secondary care in concert with cancer registry data and hospital episode statistics. Demographic data as well as information on diagnosis, histology, surgery, radiation, and previous treatment regimen were retrieved from the NO registry [32]. Effects were evaluated at first diagnosis (T0) and 6 months (T1) after first diagnosis. The occurrence of recurrences was monitored until December 2017.

2.3. Classification of Groups. Classification of groups was performed retrospectively, according to the decision of the patients to follow the advice for CTX and/or to make use of receiving add-on VA-therapy. Patients who received CTX only were allocated to the CTX-group $(n=25)$. Patients who received CTX and add-on VA were allocated to the CTX+VAgroup $(\mathrm{n}=31)$. Patients, rejecting CTX but receiving add-on VA, were allocated to the VA- group $(n=8)$. Generally, CTXand VA-therapy, when applied, started within six months after first diagnosis.

2.4. Endpoints. Patient-reported outcomes were evaluated by analyzing the ICS in all groups. The ICS is a short, highly reliable, and valid ten-item self-reported questionnaire based on a 5-point Likert scale (range: 10 (low ICS) - 50 (high ICS)). The ICS contains two subscales, one with eight items (Inner Coherence and Resilience) and a second subscale (thermo coherence) with two items. The internal consistency (Cronbach-alpha) is with $r=0.91$ robust as well as test-retestreliability (after $4-8$ weeks) is classified as good $(r=0.80)$ with sufficient validity [15]. The ICS was evaluated for T0 and T1. For the evaluation of the occurrence of recurrences the collected data of the tumor board case conferences were analyzed.

2.5. Statistical Analysis. Continuous variables were described as median with interquartile range (IQR); categorical variables were summarized as frequencies and percentages. $\mathrm{p}$ values $<0.05$ were considered to be significant. All statistical analyses were performed using the software R ( R Version 3.1.2 (2014)) [33]. For Pearson's chi-square calculation, the basic R-package was used, and for Cohen's d analyses in addition the "compute.es" package was incorporated. For all groups, baseline characteristics and treatment regimens were compared by the calculation of Pearson's chi-squares and $\mathrm{p}$ values. For further ICS-quantifications adjusted multivariable linear regression analyses were performed. Statistical models were applied for all single questions, sub- and total-scales. Predicting variables were age (in years), body mass index (BMI, continuous variable), UICC stages (categorical I, II, III), hormonal status (pre-/peri-/postmenopausal), estrogen receptor positive (yes/no), progesterone receptor positive (yes/no), and HER2 positive (yes/no).

\section{Results}

3.1. Patient Characteristics. Complete data were collected for 64 eligible women with nonmetastasized breast cancer, 
TABLE 1: Baseline characteristics of primary breast cancer patients $(n=64)$ at day of first diagnosis.

\begin{tabular}{|c|c|c|c|c|c|}
\hline & Total & CTX & CTX+VA & VA & p-value \\
\hline Number of patients, $n(\%)$ & $64(100)$ & $25(100)$ & $31(100)$ & $8(100)$ & \\
\hline Age, years, median (IQR) & $54.8(46.3-65.3)$ & $50.5(44.0-52.0)$ & $56.9(49.9-65.3)$ & $59.9(51.0-76.2)$ & 0.155 \\
\hline BMI, median (IQR) & $25.9(22.7-28.0)$ & $26.3(23.0-28.8)$ & $25.3(22.2-28.2)$ & $26.8(23.0-27.4)$ & 0.231 \\
\hline \multicolumn{6}{|l|}{ UICC stages, n (\%) } \\
\hline I & $24(37.5)$ & $9(36.0)$ & $13(41.9)$ & $2(25.0)$ & \\
\hline II & $26(40.6)$ & $10(40.0)$ & $12(38.7)$ & $4(50.0)$ & 0.926 \\
\hline III & $14(21.9)$ & $6(24.0)$ & $6(19.4)$ & $2(25.0)$ & \\
\hline \multicolumn{6}{|l|}{ Hormonal status, n (\%) } \\
\hline Pre-menopausal & $26(40.6)$ & $15(60.0)$ & $8(25.8)$ & $3(37.5)$ & \\
\hline Peri-menopausal & $5(7.8)$ & $4(16.0)$ & $1(3.2)$ & $0(0)$ & $0.008^{*}$ \\
\hline Post-menopausal & $33(51.6)$ & $6(24.0)$ & $22(71.0)$ & $5(62.5)$ & \\
\hline Estrogen receptor positive & $41(64.1)$ & $15(60.0)$ & $19(61.3)$ & $7(87.5)$ & 0.334 \\
\hline Progesteron receptor positive & $40(62.5)$ & $16(64.0)$ & $19(61.3)$ & $5(62.5)$ & 0.979 \\
\hline HER 2 positive & $23(35.9)$ & $7(28.0)$ & $14(45.2)$ & $2(25.5)$ & 0.325 \\
\hline \multicolumn{6}{|l|}{ Interventions, $\mathbf{n}(\%)$} \\
\hline CTX, n (\%) & $56(87.5)$ & $25(100)$ & $31(100)$ & $0(0)$ & $<0.001^{* *}$ \\
\hline VA therapy, n (\%) & $39(60.9)$ & $0(0)$ & $31(100)$ & $8(100)$ & $<0.001^{* *}$ \\
\hline Radiation, n (\%) & $44(68.8)$ & $19(76)$ & $22(88.0)$ & $3(37.5)$ & 0.115 \\
\hline
\end{tabular}

TNM staging according to the Union for International Cancer Control (UICC). $\mathrm{n}=$ number of patients and portion (\%). CTX = chemotherapy; VA = Viscum album L. therapy; BMI: body mass index. The p-values of Pearson's chi-square analyses of the three groups were determined using R-statistics.

which had, as a result of a multidisciplinary tumor board case conference, the advice for CTX. Medium age was 54.8 years, IQR was 46.3-65.3, and the hormonal status of $51.6 \%$ was postmenopausal; for details see Table 1. The first ICS (T0) were administered before onset of systemic anticancer therapy, usually after cancer-related surgery. The patients were assigned to the groups according to their decision, to follow the recommended CTX and/or to receive add-on VAextracts. The patients of the CTX- $(n=25)$ and CTX+VA$(\mathrm{n}=31)$ groups received CTX without or with additional VA-therapy. The 8 patients of the VA-group rejected CTX but received VA-therapy (see also study flow chart, Figure 1). Table 1 shows the main characteristics of patients of the groups at baseline. Significant differences between the groups were obtained for the patients, especially regarding their hormonal status $(\mathrm{p}=0.008)$. In the CTX-group the majority of patients were premenopausal while in the other groups most patients were postmenopausal. According to advised oncologic therapy, generally, anticancer treatments started within 6 months after first diagnosis and were administered to the patients for several years. VA-extracts of different producers, when applied, were given mostly as subcutaneous injections (for details see Table 2).

3.2. Decision-Making and ICS at First Diagnosis. The ICS was administered to all patients at first diagnosis before final decision for anticancer interventions and onset of oncological care. In Figure 2, the 10 items of the ICS are listed, and the mean values of all 10 queries were determined for the CTX, $\mathrm{CTX}+\mathrm{VA}$, and VA-groups, respectively.

In Figure 2 on the upper diagram, the profile of answer score for the three groups is shown for the mean values at T0. The total-ICS and subscores were calculated. At T0,
TABLE 2: Characteristics of VA-therapy.

\begin{tabular}{lc}
\hline & Patients, $\mathbf{n}$ \\
\hline Total number of VA patients, $\mathbf{n}(\%)$ & $39(100)$ \\
\hline Preparations, $\mathbf{n}(\%)$ & \\
$\quad$ Abnobaviscum & $24(61.5)$ \\
Iscador & $8(20.5)$ \\
Helixor & $14(35.9)$ \\
Iscucin & $4(10.3)$ \\
\hline Application, $\mathbf{n}(\%)$ & \\
subcutaneous & $33(84.6)$ \\
$\quad$ intravenous & $18(46.2)$ \\
\hline
\end{tabular}

Characteristics of VA-therapy applied additionally to standard of care (n =39). Numbers in columns do not necessarily add to 39 as patients may have received various VA combinations of preparations and applications, respectively.

before the onset of oncological care, strong and significant differences were obtained between the groups. Chi-square analyses and p-values determinations between the groups revealed significant differences for the total-ICS, the thermo coherence, and some single question scores (table in Figure 2). Demographic variables were considered to adjust these differences (Table 3). For the total-ICS the regression analysis revealed a very large and significant low total-ICS for the VAgroup (-7.521, $\mathrm{p}=0.005)$. Also the total-ICS of the CTX+VAgroup in reference to the CTX-group was lower, although not significant $(-2.691, \mathrm{p}=0.152)$. Notably, a rather low totalICS was significantly associated with the perimenopausal status $(-7.638, \mathrm{p}=0.015)$ and a higher total-ICS (although not significantly) with the postmenopausal status $(+4.705$, $\mathrm{p}=0.069)$. Moreover, the total-ICS was associated with a 


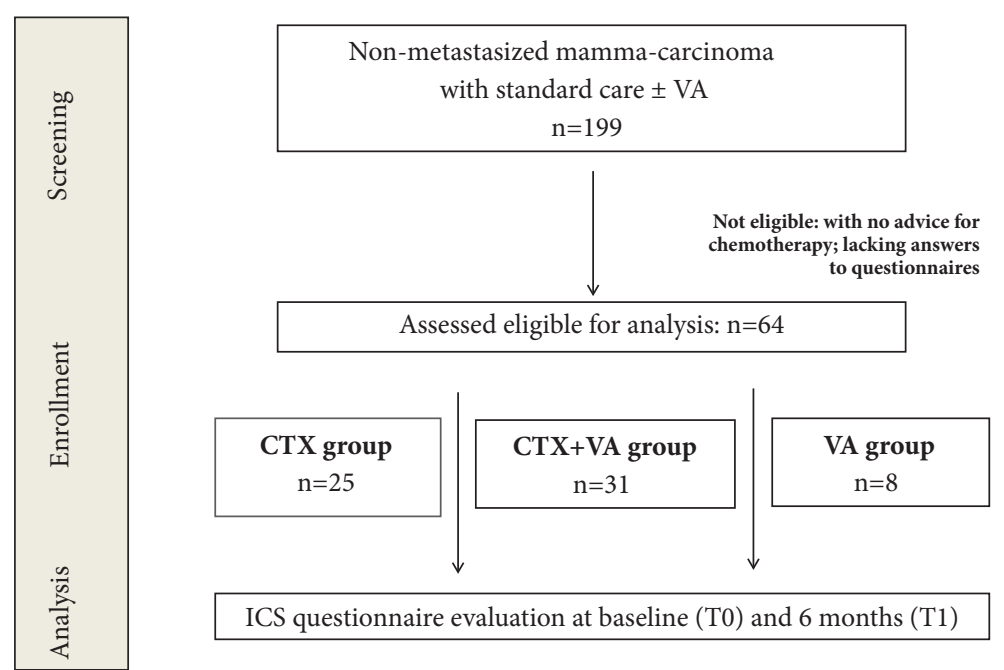

FIGURE 1: Flow chart of the study population. Classification of groups was performed according to the treatment decision of the patients. CTX-group: patients treated only with guideline-oriented CTX; CTX+VA-group: patients treated with a combination CTX and add-on VAextracts; VA-group: patients rejecting CTX but receiving add-on VA-extracts.

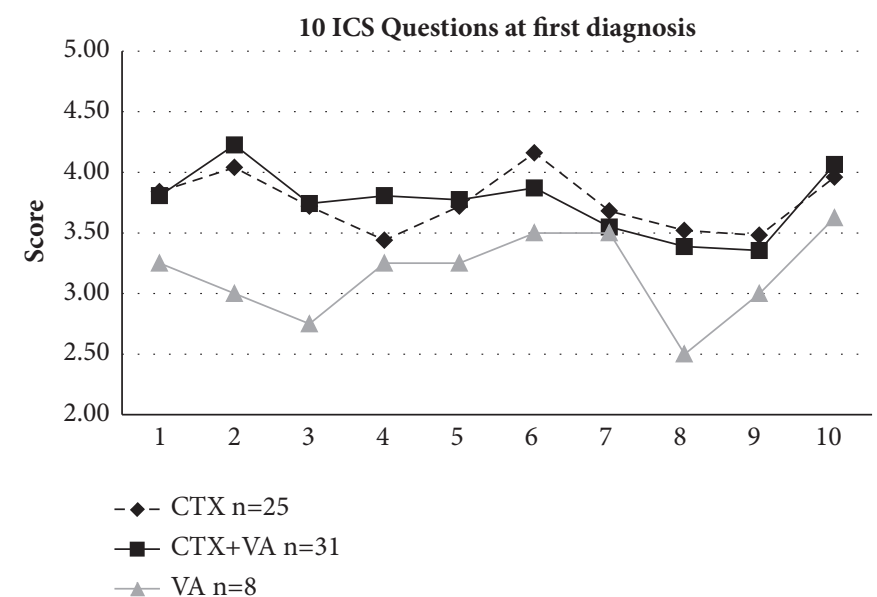

\begin{tabular}{|c|c|c|c|c|c|c|c|}
\hline \multirow{3}{*}{ Item $\quad \mathrm{n}=64$ at first diagnosis (T0) } & \multirow{2}{*}{\multicolumn{2}{|c|}{$\underbrace{}_{n=25}$}} & \multirow{2}{*}{\multicolumn{2}{|c|}{$\begin{array}{r}\mathrm{CTX}+\mathrm{VA} \\
\mathrm{n}=31\end{array}$}} & \multicolumn{2}{|c|}{ VA } & \multirow[b]{3}{*}{ p-value } \\
\hline & & & & & & $\mathrm{n}=\mathbf{8}$ & \\
\hline & Mean & SD & Mean & SD & Mean & SD & \\
\hline Total (Q1-Q10) & 37.56 & 6.11 & 37.58 & 6.22 & 31.63 & 8.97 & $0.020 *$ \\
\hline Inner Resilience Coherence (Q1+Q4 to Q10) & 29.80 & 5.71 & 29.61 & 5.39 & 25.88 & 7.46 & 0.235 \\
\hline Thermo Coherence (Q2+Q3) & 7.76 & 1.61 & 7.97 & 1.79 & 5.75 & 2.44 & $0.032 *$ \\
\hline 1) There were times last week, I felt good. & 3.84 & 0.97 & 3.81 & 0.86 & 3.25 & 1.09 & 0.228 \\
\hline 2) I felt cold without reason (inverse focusing) & 4.04 & 0.82 & 4.23 & 1.01 & 3.00 & 1.32 & $0.007 *$ \\
\hline 3) I felt pleasantly warm & 3.72 & 1.04 & 3.74 & 1.08 & 2.75 & 1.30 & 0.212 \\
\hline 4) I felt my health was... & 3.44 & 0.80 & 3.81 & 0.74 & 3.25 & 0.66 & 0.549 \\
\hline 5) I was able to face the day with confidence & 3.72 & 0.96 & 3.77 & 0.94 & 3.25 & 0.97 & 0.439 \\
\hline 6) I felt to have courage to solve problems & 4.16 & 0.83 & 3.87 & 1.04 & 3.50 & 1.41 & 0.407 \\
\hline 7) I came up with good ways to solve problems & 3.68 & 0.93 & 3.55 & 0.91 & 3.50 & 1.32 & 0.359 \\
\hline 8) what I did every day was consistent with my inner wishes & 3.52 & 0.98 & 3.39 & 1.04 & 2.50 & 1.32 & $0.002 *$ \\
\hline 9) deep down I felt secure & 3.48 & 0.81 & 3.35 & 1.03 & 3.00 & 1.22 & 0.615 \\
\hline 10) I felt moving in the right direction & 3.96 & 0.66 & 4.06 & 0.91 & 3.63 & 0.70 & 0.206 \\
\hline
\end{tabular}

FIGURE 2: ICS questionnaire of breast cancer patients. The 10 items of the ICS questionnaire with answer possibilities $1-5(1=$ low ICS, $5=$ high ICS). In the upper diagram the profile of answer score of all 10 items for all groups is shown for the values at first diagnosis. Subscales, mean values, standard deviations, and p-values were calculated. 
TABLE 3: Association factors for ICS and subscores.

\begin{tabular}{|c|c|c|c|c|c|c|c|c|c|}
\hline \multirow{2}{*}{$\mathrm{n}=64$} & \multicolumn{3}{|c|}{ Total ICS } & \multicolumn{3}{|c|}{ Inner resilience coherence } & \multicolumn{3}{|c|}{ Thermo coherence } \\
\hline & estimate & SE & p-value & estimate & SE & p-value & estimate & SE & p-value \\
\hline \multicolumn{10}{|c|}{ demographic variables } \\
\hline Age & -0.135 & 0.092 & 0.147 & -0.099 & 0.081 & 0.226 & -0.053 & 0.027 & 0.055 \\
\hline BMI & -0.384 & 0.159 & $0.019^{*}$ & -0.362 & 0.142 & $0.014^{*}$ & -0.035 & 0.047 & 0.458 \\
\hline \multicolumn{10}{|l|}{ Hormonal status } \\
\hline Pre-menopausal & reference & & & reference & & & reference & & \\
\hline Peri-menopausal & -7.638 & 3.037 & $0.015^{*}$ & -6.930 & 2.716 & $0.013^{*}$ & -0.357 & 0.903 & 0.694 \\
\hline Post-menopausal & 4.705 & 2.536 & 0.069 & 3.604 & 2.237 & 0.112 & 1.507 & 0.707 & $0.037^{*}$ \\
\hline Group & & & & & Decision & & & Decision & \\
\hline Reference & & CTX & & & no VA- & & & 1о CTX- & \\
\hline CTX+VA & -2.691 & 1.852 & 0.152 & -3.076 & 1.580 & 0.056 & 2.000 & 0.697 & $0.006^{* *}$ \\
\hline VA & -7.521 & 2.574 & $0.005^{* *}$ & & VA-therapy & & & CTX & \\
\hline
\end{tabular}

Multivariable linear regression analyses using R-statistics were performed for decision making and the total-ICS, the inner resilience coherence, and thermo coherence at T0. CTX group: patients treated only with guideline-oriented CTX; CTX+VA group: patients treated with a combination CTX and add-on VAextracts; VA-group: patients rejecting CTX but receiving add-on VA-extracts.

TABLE 4: Association factors for ICS single scores.

\begin{tabular}{|c|c|c|c|c|c|c|c|c|c|}
\hline \multirow[t]{2}{*}{$n=64$} & \multicolumn{3}{|c|}{$\begin{array}{l}\text { Question 2: } \\
\text { I felt cold without reason }\end{array}$} & \multicolumn{3}{|c|}{$\begin{array}{l}\text { Question 6: } \\
\text { Courage to solve problems }\end{array}$} & \multicolumn{3}{|c|}{$\begin{array}{l}\text { Question 8: Consistency } \\
\text { with my inner wishes }\end{array}$} \\
\hline & estimate & SE & p-value & estimate & SE & p-value & estimate & SE & p-value \\
\hline \multicolumn{10}{|c|}{ demographic variables } \\
\hline Age & -0.042 & 0.015 & $0.008^{* *}$ & -0.027 & 0.016 & 0.09 & 0.005 & 0.016 & 0.762 \\
\hline BMI & -0.014 & 0.026 & 0.591 & -0.033 & 0.026 & 0.225 & -0.03 & 0.027 & 0.277 \\
\hline \multicolumn{10}{|l|}{ Hormonal status } \\
\hline Pre-menopausal & reference & & & reference & & & reference & & \\
\hline Peri-menopausal & -0.021 & 0.508 & 0.968 & -1.115 & 0.519 & $0.036^{*}$ & -1.300 & 0.536 & $0.019^{*}$ \\
\hline Post-menopausal & 1.132 & 0.42 & $0.009^{* *}$ & 0.645 & 0.43 & 0.139 & -0.072 & 0.444 & 0.872 \\
\hline Receptor negative & reference & & & reference & & & reference & & \\
\hline Estrogen positive & -0.204 & 0.377 & 0.59 & -0.139 & 0.385 & 0.72 & 1.068 & 0.4 & $0.010^{*}$ \\
\hline Prog positive & 0.278 & 0.361 & 0.445 & 0.002 & 0.369 & 0.996 & -0.697 & 0.381 & 0.073 \\
\hline HER2 positive & 0.163 & 0.275 & 0.555 & 0.171 & 0.281 & 0.547 & 0.338 & 0.291 & 0.249 \\
\hline \multicolumn{10}{|l|}{ Decision } \\
\hline $\mathrm{CTX}^{1}$ & 0.903 & 0.408 & $0.031^{*}$ & 0.153 & 0.418 & 0.715 & 1.108 & 0.431 & $0.013^{*}$ \\
\hline $\mathbf{V A}^{2}$ & -0.114 & 0.302 & 0.707 & -0.623 & 0.309 & $0.049^{*}$ & -0.417 & 0.319 & 0.197 \\
\hline
\end{tabular}

Multivariable regression analyses for the single questions 2 (I felt cold without reason), 6 (courage to solve problems), and 8 (consistent with my inner wishes). References for treatment decisions were ${ }^{1}$ no CTX or ${ }^{2}$ no VA-therapy.

lowered BMI $(-0.384, \mathrm{p}=0.019)$. In further multivariable regression analyses in Table 3 , age, BMI, and hormonal status were considered and instead of the group, the decisions for CTX or VA-therapy, respectively, were included as variables. There was a negative association between the inner resilience and coherence subscale, and the decision for VA-therapy was nearly significant $(-3.076, \mathrm{p}=0.056)$. Similar as for the totalICS, the inner resilience and coherence were significantly associated with a lowered BMI and with a perimenopausal status. Furthermore, an increased thermo coherence score was significantly associated with the decision for CTX. With regard to the hormonal status, a postmenopausal status was significantly associated with a higher thermo coherence score $(+1.507, \mathrm{p}=0.037)$ while no considerable thermo coherence differences were obtained between pre- and perimenopausal patients. Among the means of the scores for single questions, significant differences between the groups were obtained for question 2 (feeling cold; $\mathrm{p}=0.007$ ) and question 8 (what I did every day was consistent with my inner wishes; $p=0.002$ ) (see Figure 2). In Table 4 multivariable regression analyses for scores of single questions, considering age, BMI, hormonal status, and decision for CTX or VA-therapy, respectively, are shown. High scores for question 2 (this means, rarer feeling cold without reason) were significantly associated with the decision for CTX $(+0.903$ with $\mathrm{p}=0.031)$ and also for the postmenopausal status $(+1.132, \mathrm{p}=0.009)$. Furthermore, the decision for CTX was associated with a significant high score for question 8 (consistency with my inner wishes) $(+1.108, \mathrm{p}=0.013)$. In addition, further striking differences were obtained. In particular, a low score for question 6 (this 


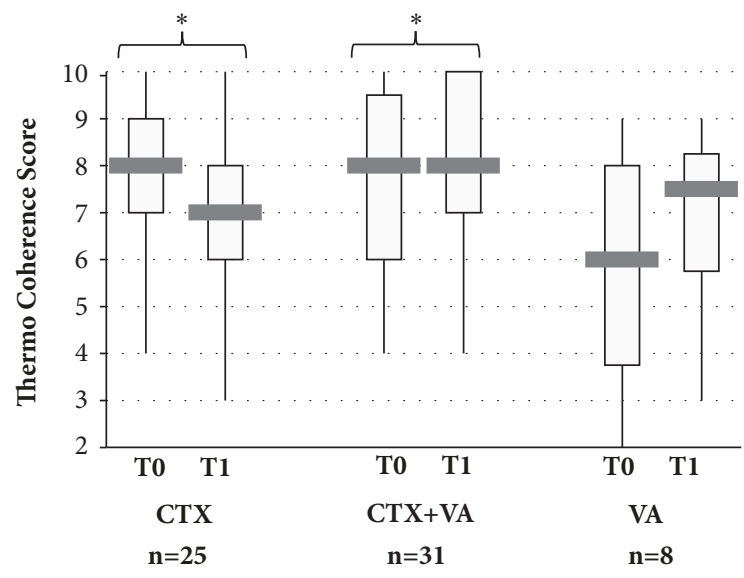

FIGURE 3: Thermo coherence during therapy of breast cancer patients. Boxplots for the thermo coherence scores for the CTX-, $\mathrm{CTX}+\mathrm{VA}$, and VA-group at first diagnosis (T0) and 6 months later (T1). * Calculation of Cohen's d revealed for the CTX $d[95 \% \mathrm{CI}]=$ $0.70[0.09,1.31], \mathrm{p}(\mathrm{d})=0.02^{*}$ and for the CTX+VA-group $d[95 \%$ $\mathrm{CI}]=0.96[0.39,1.53], \mathrm{p}(\mathrm{d})<0.01$.

means, rarer having courage to solve problems) seemed to be associated with a perimenopausal status (-1.115 with $\mathrm{p}$ $=0.036)$ and also for the decision for VA-therapy $(-0.623$, $0.049)$.

3.3. Thermo Coherence. In Figure 3 boxplots for the thermo coherence for all groups at first diagnosis (T0) and 6 months of follow-up (T1) are shown. For the CTX-group the thermo coherence score decreased significantly during chemotherapy. Pearson's chi-squared test and Cohen's d analyses revealed a significant medium effect size, $d$ [95\% CI] $=0.70[0.09,1.31], \mathrm{p}(\mathrm{d})=0.02$ for this decrease. In contrast, for the other groups increased thermo coherence scores were detected during VA-therapy (Figure 3). For the CTX+VAgroup a significant effect was obtained, $d[95 \% \mathrm{CI}]=0.96$ $[0.39,1.53], p(d)<0.01$ for this increase. Similarly, an increase of the thermo coherence score was obtained for the VAgroup; however, due to the small sample size these analyses were not yet statistically significant $(d[95 \% \mathrm{CI}]=0.99[-0.25$, $2.24], \mathrm{p}(\mathrm{d})=0.11)$.

3.4. Occurrence of Recurrences. The first diagnoses of the entire study population $(n=64)$ were carried out between March 2012 and February 2017. For a three-year follow-up evaluation of the occurrence of tumor recurrences only the patients $(n=41)$ with first diagnosis before December 2014 were taken into consideration (14 CTX, $21 \mathrm{CTX}+\mathrm{VA}$, and 6 VA). Until December 2017 in 8 from 41 cases (19.5\%), recurrences were detected within 13- 40 months after their first diagnosis (Figure 4). The rates of occurrence of recurrences in the CTX and in the CTX+VA-groups were comparable, about $14 \%$ ( 2 in the CTX-group and 3 in the CTX+VA-group), while in 3 patients of the CTX rejecting group (VA-group) recurrences were detected $(50 \%)$. Of the 8 patients with recurrences, 3 of them rejected CTX and radiation, 3 have had UICC-Class I, 4 have had UICC-Class II, and 1 has had UICC-Class III tumors, and 6 of them were HER2 positive. 3 patients were premenopausal, 4 were postmenopausal, and 1 was perimenopausal.

\section{Discussion}

The results of the present study reveal that the individual inner coherence and hormonal status of breast cancer patients seem to be crucial for decision finding for cancer therapy. Strikingly, low scores for consistency with inner wishes and thermo coherence were associated with the decision against CTX. On the other side, a low score of the inner resilience coherence, especially rarer having courage to solve problems, is significantly associated with the decision for an additional VA-therapy. Furthermore, for patients treated additionally with VA-extracts, their thermo coherence scores increased significantly 6 months after first diagnosis.

At time of first diagnosis, before patients decided for the respective oncological treatment, common features as well as significant differences in the individual ICS-profiles were observed, although these ICS-queries were not integrated in the decision-finding process. As being reported previously, the ICS is associated with gender and age [15]. In line with this finding, we observed significant associations between the hormonal status and the ICS of the patients in the present study. For the perimenopausal patients the scores of the inner resilience and coherence were significantly low, while the thermo coherence of postmenopausal patients was significantly high. A thermal dysregulation has been seen particularly in premenopausal breast cancer patients and can be related to explicitly affect the HRQL [34]. Notably, CTX increases fatigue levels [35] and has been linked to "feeling cold" [17]. Moreover, the symptoms, such as "feeling cold" or hot flushes and congestive sweating, have been discussed as a constitutional pattern in premenopausal breast cancer patients [34]. In line with these expectations, a significant decline of the thermo coherence score in the present study was found 6 months after onset of CTX but only for the CTX-group (Figure 3). In contrast, the thermo coherence scores of the other groups which were treated with VAextracts increased, suggesting that VA-applications might support thermoregulation in breast cancer patients and this may in part alleviate thermal discomfort. Reduced internal coherence scale values in association with loss of autonomic, rest/activity, and orthostatic-circulatory regulation in breast cancer patients were reported in another case-control study [36].

The rates of occurring recurrences in the CTX-related to the CTX+VA-group were comparable and at present date, in $50 \%$ of patients rejecting CTX towards just $14 \%$ of patients receiving CTX, developing recurrences were detected. Even if these preliminary analyses did not yield significant group differences between the recurrence rates, the necessity of undergoing CTX should not be called into question. Thus, there is an urgent need to encourage patients rejecting CTX and to offer them an efficient treatment regimen by reducing adverse effects to a bearable extent.

CTX is known to negatively affect HRQL [35]. Clinician's recommendations play a significant role in either accepting or declining CTX. For instance, Harder et al. 


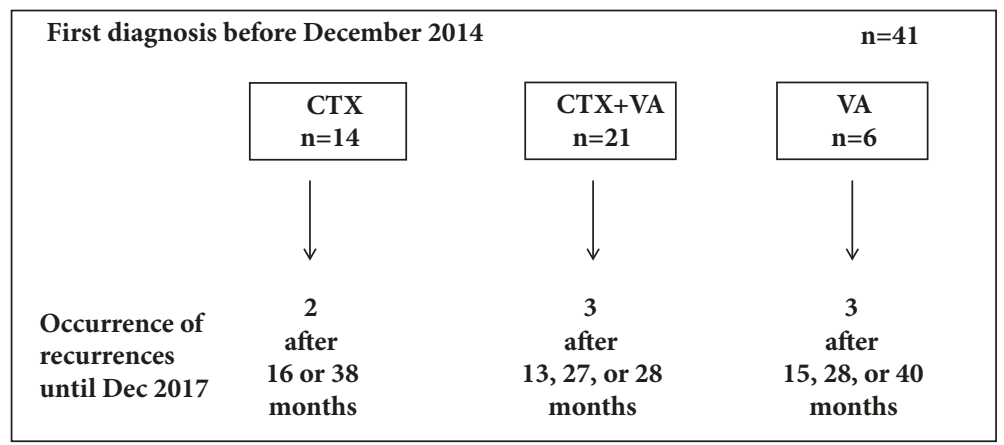

FIGURE 4: Three-year follow-up of tumor recurrences. The occurrence of tumor recurrences was evaluated for the patients $(\mathrm{n}=41)$ with first diagnosis before December 2014.

[37] reported that older women with early breast cancer preferred to be involved in clinical decision-making. Medical oncologists should be able to apply decision aid modalities in a personalized manner to give all needed information to their patients, thereby ensuring a deliberate shared decisionmaking process [4] and facilitating acceptance of a need for CTX. Thus, any treatment implemented should be in harmony with the patient's preferences [38]. Currently, there is no guideline for the treatment of patients who reject CTX. In the certified Breast Cancer Center of the present study, informed decision-making is performed according to the German S3-guideline for early detection, diagnosis, therapy, and follow-up of breast cancer [3] but it also takes into account the individual life situation and needs of the patients.

\section{Conclusions}

In the present analysis it is shown that patients with a low internal coherence, especially thermo coherence, tend to reject immediate CTX. On the other side, it is demonstrated that VA-applications led to a better thermoregulation. Thus add-on VA might present a decision option, to encourage patients to undergo CTX, at least at a later time, to ensure prevention of progress of tumor development.

\section{Data Availability}

The datasets used and/or analyzed during the current study have been kept confidential and are not available publicly.

\section{Conflicts of Interest}

The Network Oncology was partially funded by Iscador AG Arlesheim, Switzerland, Abnoba GmbH Pforzheim, Germany, and Helixor Heilmittel GmbH Rosenfels, Germany. By contract, researchers were independent from the funder. Friedemann Schad and Matthias Kröz received honoraria for lectures from Helixor Heilmittel $\mathrm{GmbH}$ outside the submitted work. All other authors declare that they have no conflicts of interest.

\section{Authors' Contributions}

Shiao Li Oei and Anja Thronicke contributed equally to this work.

\section{Acknowledgments}

We would like to thank all medical documentation officers at the GKH and the FIH involved in the present work.

\section{References}

[1] R. L. Siegel, K. D. Miller, and A. Jemal, "Cancer Statistics, 2017," CA: A Cancer Journal for Clinicians, vol. 67, no. 1, pp. 7-30, 2017.

[2] M. Kenyon, D. K. Mayer, and A. K. Owens, "Late and long-term effects of breast cancer treatment and surveillance management for the general practitioner," JOGNN - Journal of Obstetric, Gynecologic, and Neonatal Nursing, vol. 43, no. 3, pp. 382-398, 2014.

[3] D. Krebsgesellschaft and D. Krebshilfe, "Arbeitsgemeinschaft der Wissenschaftlich Medizinischen Fachgesellschaften," Interdisziplinäre S3-Leitlinie für die Diagnostik, Therapie und Nachsorge des Mammakarzinoms, vol. 2017, 2017, http://www.leitlinienprogramm-onkologie.de/index.php.

[4] A. M. Berger, R. J. Buzalko, K. A. Kupzyk, B. J. Gardner, D. M. Djalilova, and J. L. Otte, "Preferences and actual chemotherapy decision-making in the greater plains collaborative breast cancer study*”, Acta Oncologica, vol. 56, no. 12, pp. 1690-1697, 2017.

[5] G. A. Kelley, K. S. Kelley, and E. Shimizu, "Meditative Movement Therapies and Health-Related Quality-of-Life in Adults: A Systematic Review of Meta-Analyses," PLoS ONE, vol. 10, no. 6, p. e0129181, 2015.

[6] A. Lipsett, S. Barrett, F. Haruna, K. Mustian, and A. O’Donovan, "The impact of exercise during adjuvant radiotherapy for breast cancer on fatigue and quality of life: A systematic review and meta-analysis," The Breast, vol. 32, pp. 144-155, 2017.

[7] K. Shimozuma, T. Okamoto, N. Katsumata et al., "Systematic overview of quality of life studies for breast cancer," Breast Cancer, vol. 9, no. 3, pp. 196-202, 2002.

[8] N. K. Aaronson, S. Ahinedzai, B. Bergman et al., “The European Organization for Research and Treatment of Cancer QLQ-C30: a quality-of-life instrument for use in international clinical trials in oncology," Journal of the National Cancer Institute, vol. 85, no. 5, pp. 365-376, 1993. 
[9] D. F. Cella, D. S. Tulsky, G. Gray et al., "The functional assessment of cancer therapy scale: development and validation of the general measure," Journal of Clinical Oncology, vol. 11, no. 3, pp. 570-579, 1993.

[10] A. Antonovsky, "The structure and properties of the sense of coherence scale," Social Science \& Medicine, vol. 36, no. 6, pp. 725-733, 1993.

[11] R. Grossarth-Maticek, Systemische Epidemiologie und präventive Verhaltensmedizin chronischer Erkrankungen, Walter de Gruyter, Berlin, Boston, 1999.

[12] A. Büssing, M. Girke, C. Heckmann, F. Schad, T. Ostermann, and M. Kröz, "Validation of the self regulation questionnaire as a measure of health in quality of life research," European Journal of Medical Research, vol. 14, no. 5, pp. 223-227, 2009.

[13] R. Grossarth-Maticek and R. Ziegler, "Prospective controlled cohort studies on long-term therapy of breast cancer patients with a mistletoe preparation (Iscador)," Forschende Komplementärmedizin, vol. 13, no. 5, pp. 285-292, 2006.

[14] M. Eriksson and B. Lindström, “Antonovsky's sense of coherence scale and its relation with quality of life: a systematic review," Journal of Epidemiology and Community Health, vol. 61, no. 11, pp. 938-944, 2007.

[15] M. Kröz, A. Büssing, H. B. von Laue et al., "Reliability and validity of a new scale on internal coherence (ICS) of cancer patients," Health and Quality of Life Outcomes, vol. 7, article no. 59, 2009.

[16] S. M. Frank, S. N. Raja, C. F. Bulcao, and D. S. Goldstein, "Relative contribution of core and cutaneous temperatures to thermal comfort and autonomic responses in humans," Journal of Applied Physiology, vol. 86, no. 5, pp. 1588-1593, 1999.

[17] K. M. Kokolus, C.-C. Hong, and E. A. Repasky, "Feeling too hot or cold after breast cancer: is it just a nuisance or a potentially important prognostic factor?" International Journal of Hyperthermia, vol. 26, no. 7, pp. 662-680, 2010.

[18] M. Kröz, M. Reif, R. Zerm et al., "Mistletoe and chemotherapy responsiveness of different scales in oncological patients undergoing chemotherapy," Phytomedicine, vol. 18, p. S16, 2011.

[19] M. Horneber, G. Bueschel, G. Dennert, D. Less, E. Ritter, and M. Zwahlen, "How many cancer patients use complementary and alternative medicine: a systematic review and metaanalysis," Integrative Cancer Therapies, vol. 11, no. 3, pp. 187-203, 2012.

[20] M. Baum, B. R. Cassileth, R. Daniel et al., "The role of complementary and alternative medicine in the management of early breast cancer: Recommendations of the European Society of Mastology (EUSOMA)," European Journal of Cancer, vol. 42, no. 12, pp. 1711-1714, 2006.

[21] G. S. Kienle, A. Glockmann, M. Schink, and H. Kiene, "Viscum album L. extracts in breast and gynaecological cancers: a systematic review of clinical and preclinical research," Journal of Experimental \& Clinical Cancer Research, vol. 28, no. 1, article 79, 2009.

[22] G. S. Kienle, R. Grugel, and H. Kiene, "Safety of higher dosages of Viscum album L. in animals and humans-systematic review of immune changes and safety parameters," BMC Complementary and Alternative Medicine, vol. 11, article 72, 2011.

[23] M. A. Horneber, G. Bueschel, R. Huber, K. Linde, and M. Rostock, "Mistletoe therapy in oncology," Cochrane Database of Systematic Reviews, no. 2, 2008.

[24] M. L. Steele, J. Axtner, A. Happe, M. Kröz, H. Matthes, and F. Schad, "Adverse drug reactions and expected effects to therapy with subcutaneous mistletoe extracts (Viscum album
L.) in cancer patients," Evidence-Based Complementary and Alternative Medicine, vol. 2014, 2014.

[25] M. L. Steele, J. Axtner, A. Happe, M. Kröz, H. Matthes, and F. Schad, "Use and safety of intratumoral application of European mistletoe (Viscum album L) preparations in oncology," Integrative Cancer Therapies, vol. 14, no. 2, pp. 140-148, 2015.

[26] M. Kröz, F. Schad, B. Matthes, H. Pickartz, and M. Girke, "Blood and tissue eosinophilia, mistletoe lectin antibodies and quality of life in a breast cancer patient undergoing intratumoral and subcutaneous mistletoe injection," Forschende Komplementärmedizin und klassische Naturheilkunde, vol. 9, no. 3, pp. 160-167, 2002.

[27] V. F. Semiglasov, V. V. Stepula, A. Dudov, W. Lehmacher, and U. Mengs, "The standardised mistletoe extract PS76A2 improves QoL in patients with breast cancer receiving adjuvant CMF chemotherapy: a randomised, placebo-controlled, double-blind, multicentre clinical trial," Anticancer Reseach, vol. 24, no. 2, pp. 1293-1302, 2004.

[28] V. F. Semiglazov, V. V. Stepula, A. Dudov, J. Schnitker, and U. Mengs, "Quality of life is improved in breast cancer patients by standardised mistletoe extract PS76A2 during chemotherapy and follow-up: a randomised, placebo-controlled, double-blind, multicentre clinical trial," Anticancer Reseach, vol. 26, no. 2, pp. 1519-1529, 2006.

[29] J. Eisenbraun, R. Scheer, M. Kröz, F. Schad, and R. Huber, "Quality of life in breast cancer patients during chemotherapy and concurrent therapy with a mistletoe extract," Phytomedicine, vol. 18, no. 2-3, pp. 151-157, 2011.

[30] F. Schad, A. Thronicke, A. Merkle et al., "Implementation of an Integrative Oncological Concept in the Daily Care of a German Certified Breast Cancer Center," Complementary Medicine Research, vol. 25, no. 2, pp. 85-91, 2018.

[31] A. Thronicke, M. Kröz, A. Merkle, H. Matthes, C. Herbstreit, and F. Schad, "Psychosocial, Cognitive, and Physical Impact of Elaborate Consultations and Life Review in Female Patients with Non-Metastasized Breast Cancer," Complementary Medicine Research, vol. 25, no. 2, pp. 92-101, 2018.

[32] F. Schad, J. Axtner, A. Happe et al., "Network Oncology (NO) - A Clinical Cancer Register for Health Services Research and the Evaluation of Integrative Therapeutic Interventions in Anthroposophic Medicine," Forschende Komplementärmedizin, vol. 20, no. 5, pp. 353-360, 2013.

[33] Team RC, R: A language and environment for statistical computing, R Foundation for Statistical Computing, Vienna, Austria, 2016.

[34] M. Kröz, C. Heckmann, and M. Weckenmann, "Development of body weight, vegetative nervous system, and breast cancer," Forschende Komplementärmedizin und klassische Naturheilkunde, vol. 7, no. 3, pp. 132-138, 2000.

[35] A. M. Berger, K. Lockhart, and S. Agrawal, "Variability of patterns of fatigue and quality of life over time based on different breast cancer adjuvant chemotherapy regimens," Oncology Nursing Forum, vol. 36, no. 5, pp. 563-570, 2009.

[36] J. Linke, Cancer Fatigue und gestörte Ruhe/Aktivitäts-Regulation bei Mammakarzinom-Patientinnen [Dissertation, thesis], 2008, http://www.diss.fu-berlin.de/diss/receive/FUDISS_thesis_ 000000006970.

[37] H. Harder, R. Ballinger, C. Langridge, A. Ring, and L. J. Fallowfield, "Adjuvant chemotherapy in elderly women with breast cancer: patients' perspectives on information giving and decision making," Psycho-Oncology, vol. 22, no. 12, pp. 27292735, 2013. 
[38] S. J. T. Jansen, W. Otten, and A. M. Stiggelbout, "Factors affecting patients' perceptions of choice regarding adjuvant chemotherapy for breast cancer," Breast Cancer Research and Treatment, vol. 99, no. 1, pp. 35-45, 2006. 


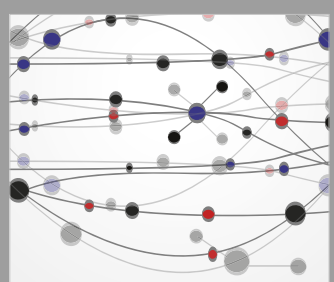

The Scientific World Journal
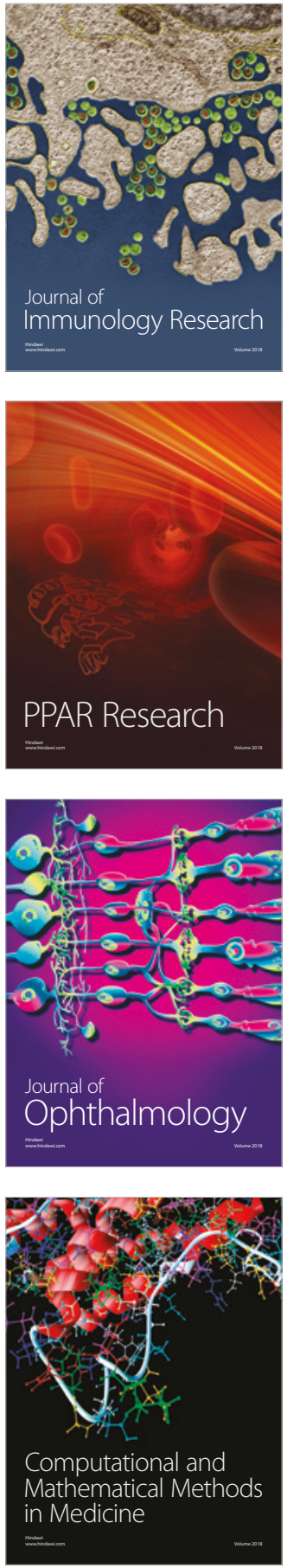

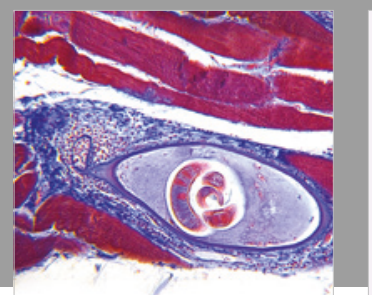

Gastroenterology Research and Practice

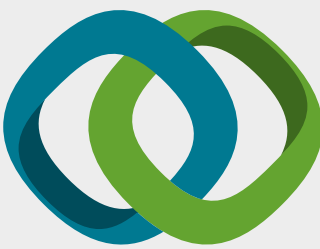

\section{Hindawi}

Submit your manuscripts at

www.hindawi.com
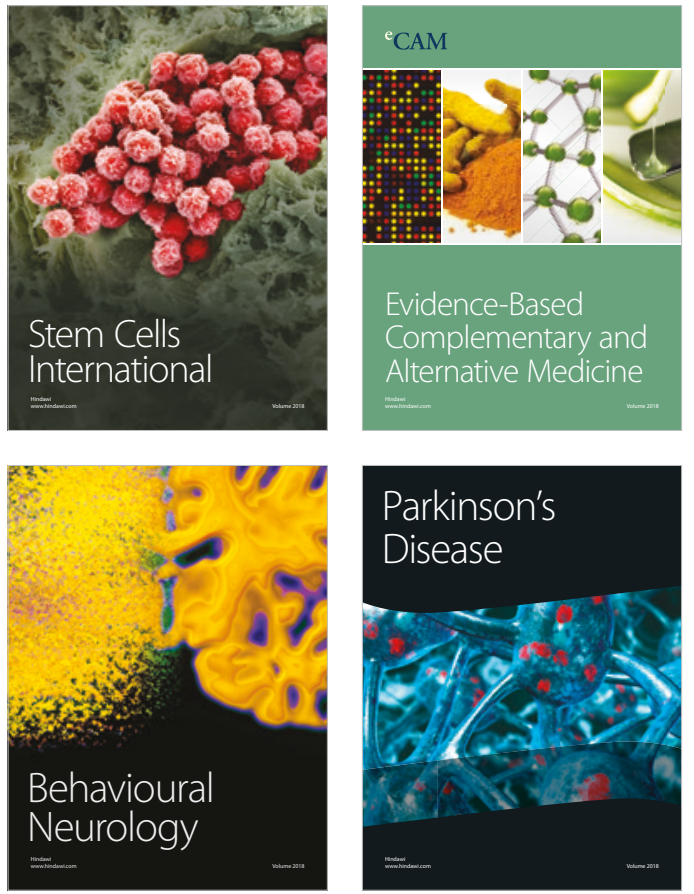

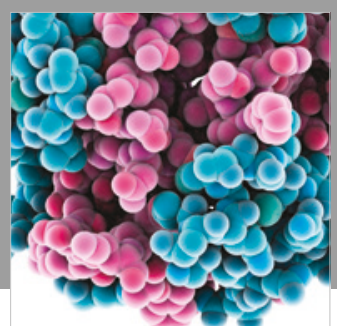

ournal of

Diabetes Research

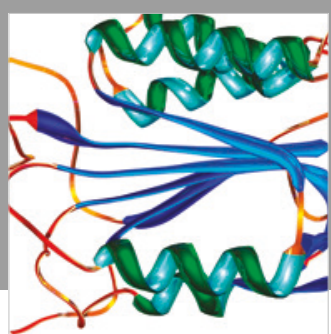

Disease Markers
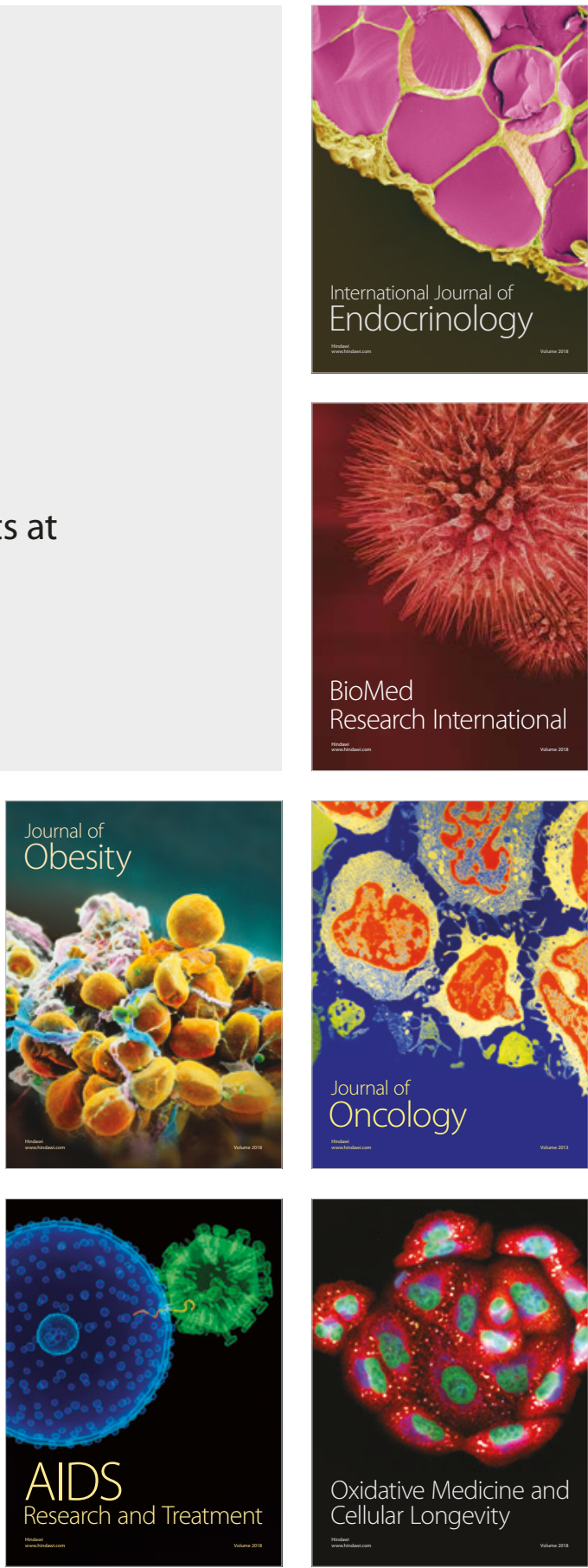